\section{AL-AZHAR Dental Journal}

F o r
The Official Publication of The Faculty of Dental Medicine For Girls, Al-Azhar University Cairo, Egypt.

Print ISSN 2537-0308 • Online ISSN 2537-0316

ADJ-for Girls, Vol. 7, No. 4, October (2020) - PP. 521:529

\title{
Evaluation of the Clinical Performance of Reinforced Glass Ionomer Restorations and Influence of Heat Application on Their Shear Bond Strength and Microleakage
}

\author{
Nessma M. Mahmoud ${ }^{1 *}$, Maha A. Niazy ${ }^{2}$ and Mohamed A. Elyasaky ${ }^{3}$
}

\begin{tabular}{|l|}
\hline Codex : 65/20.10 \\
azhardentj@ azhar.edu.eg \\
http://adjg.journals.ekb.eg \\
\hline DOI: $10.21608 /$ adjg.2020.13541.1166 \\
\hline $\begin{array}{l}\text { Restorative Dentistry } \\
\text { Removable Prosthodontics, Fixed } \\
\text { Prosthodontics, Endodontics, Dental } \\
\text { Biomaterials, Operative Dentistry) }\end{array}$ \\
\hline
\end{tabular}

KEYWORDS

Glass ionomer,

Shear bond strength,

Microleakage

\begin{abstract}
Purpose: This study evaluated and compared between effect of heat application on the shear bond strength and microleakage of class I reinforced glass ionomer restorations (EquiaFil Fort, Zirconomer and Resin modified glass ionomer Fuji II LC). Meanwhile, the clinical performance of such materials was assessed. Materials and Methods: Ninety caries free first and second permanent molars were divided into three groups of (30 each) according to the type of the Glass Ionomer Cements used. Each group was further subdivided into three subgroups of (10 each): $B_{1}$ for no heat application, $\mathrm{B}_{2}$ for $20 \mathrm{sec}$ heat application and $\mathrm{B}_{3}$ for $60 \mathrm{sec}$ heat application. This sample grouping was done twice in shear bond strength and microleakage assessment. A universal testing machine was used to test the shear bond strength for the specimens, while the microleakage test was examined under stereomicroscope. A total of 20 patients with split mouth design participated in this study. Each patient had two fillings according to the material used (Equia and Zirconomer). Restorations of the teeth were evaluated at the same day (base line), one month, and 6 months and were rated according to the USPHS criteria. Results: all heated groups showed higher shear bond strength and microleakage. For secondary caries, post-operative sensitivity and color match, no statistical significance differences were recorded in the clinical performance of Equia and Zirconomer. Conclusion: Application of heat improves bond strength and microleakage of GICs. The clinical outcome of both Equia and Zirconomer were considered satisfactory after 6 months.
\end{abstract}

- Paper extracted from Doctor thesis titled “Evaluation of the Clinical Performance of Reinforced Glass Ionomer Restorations and Influence of Heat Application on Their Shear Bond Strength and Microleakage “

1. MD of Operative Dentistry, Faculty of Dental Medicine for Girls, Al-Azhar University, Cairo, Egypt

2. Professor of Operative Dentistry, Faculty of Dental Medicine for Girls, Al-Azhar University, Cairo, Egypt

3. Professor of Operative Dentistry \& Dean of the Faculty of Dental Medicine, Sinai University

* Corresponding author email: nesma_sedky@yahoo.com 


\section{INTRODUCTION}

Nowadays, the advanced Operative dentistry concentrated on minimal tooth tissue removal and focused on developing the adhesive restorative materials that have remineralizing action on demineralized dentin. Those requirements were found in glass ionomer cements (GICs) ${ }^{(1)}$.

Many trials have been done to modify these materials for years. Their main advantages are adhesion to tooth structure, anticariogenic properties due to the flouride discharge and biocompatibility but they also lack high fracture toughness and have higher rate of occlusal wear than amalgam and composite $^{(2)}$.

Recently, a newer GI material Zirconomer which has been reinforced with zirconia fillers has been introduced to combat the disadvantages of composites and amalgams. It is known to exhibit the strength and durability of amalgam with the advantages of GICs without the mercury threats ${ }^{(3)}$.

Highly viscous GICs have a high cross-linkage GICs matrix leading to higher physical properties when compared to traditional GICs. Equia, a new GIC has been innovated consisting of a high viscous conventional GIC (EquiaFil, known before as Fuji IX GP Extra) with a new nanofilled coating material (Equia Coat, known before as G-Coat Plus) ${ }^{(4)}$. This nanofilled hydrophilic resin coat having a very low viscosity provides the GIC surface a very good seal. Nanofillers protect the whole restoration against abrasive wear especially in the first month until the GIC is completely mature and can resist the intraoral stresses. The coating also acts as a glaze, so accepted esthetically ${ }^{(5)}$.

Several researches have tried to start the set of conventional GICs using ultrasonic treatment (6) or heat utilization. Rapid setting allows for shorter clinical time and better clinical procedures ${ }^{(7)}$.

Accordingly, we aimed in this study to heat the reinforced GICs, then investigate its effect on their shear bond strength and their microleakage. In addition, their clinical performance was evaluated invivo.

\section{MATERIALS AND METHODS}

\section{1- Teeth selection:}

A total of one hundred and eighty caries free first and second permanent molars extracted for periodontal causes were collected, then cleaned of any soft tissues and calculus using hand scalers then checked by magnifying loupes to be sure that they were free of any cracks, caries or other defects. They were stored in glass container containing normal saline and used within one month.

\section{2- Samples' grouping:}

Samples were divided into three groups of (30) teeth according to the type of the material used. Group A1: Equia Forte Fil, Group A2: Zirconomer Improved and Group A3: Fuji II LC. Further subdivision of each group into three subgroups, each contains (10 samples each) B1: no heat application, B2: $20 \mathrm{sec}$ heat application and B3: $60 \mathrm{sec}$ heat application. This sample grouping was done twice for shear bond strength and microleakage assessment. Ninety teeth for each test.

\section{3- Microleakage assessment:}

Class I cavities were prepared using straight fissured and inverted cone diamond burs so that the cavity dimensions was $4 \mathrm{~mm}$ wide, $2 \mathrm{~mm}$ deep and $4 \mathrm{~mm}$ long. A graduated periodontal probe was used to determine the cavity dimensions. In the first, second and third groups, thirty molars were restored by Equia Forte fil, Zirconomer Improved and Fuji II LC respectively following the manufacturer's protocol, then they were sub divided into three subgroups where the first subgroup was not subjected to heat application while second subgroup and the third one were subjected to 20 and 60 seconds of heat application respectively. A light cure unit (420$480 \mathrm{~nm}$ ) was used to apply heat at 0 distance. One layer of nail varnish was applied on all teeth surfaces with exception of the restoration and $1 \mathrm{~mm}$ band around the periphery of the restorations and left to dry. A second layer was applied in the same manner 
to allow for proper sealing. The apices of the roots were sealed using composite.

Teeth were immersed in a freshly prepared aqueous methylene blue solution with a concentration of $2 \mathrm{gm} / 200 \mathrm{c}$.c water for 24 hours at room temperature. After removing the samples from the dye, they were washed under running water for 3 minutes to remove any remain dye. A diamond disc was used to vertically divide the teeth through the center of the GIC fixed in a cutting machine. The sectioned specimens were examined under stereomicroscope at $35 \mathrm{x}$ magnification.

\section{4- Shear bond strength assessment:}

The ninety samples were washed in tap water to remove the remaining storage solution, crowns were separated from the roots at about $0.5 \mathrm{~mm}$ apical to cemeno-enamel junction using a diamond disc in low speed straight hand piece under constant water spray. Crowns were bisected transversally in a buccolingual direction, specially fabricated split copper cubic mold of $1.5 \mathrm{~cm}$ length was used. The specimens were put deeply in self cured acrylic resin (Acrostone) inside the mold with their dentin surfaces upward. Demarcation of the bonding site was done to define a fixed test surface of $3 \mathrm{~mm}$ diameter) using an adhesive tape, so that the bond strength recorded would only be related to the predefined area. A specially fabricated metal mold enclosing a split acrylic disc having a mid hole of $3 \mathrm{~mm}$ in diameter and $2 \mathrm{~mm}$ in height was used to help material packing. In the first, second and third groups, thirty molars were restored by Equia Forte fil, Zirconomer Improved and Fuji II LC respectively according to the manufacturer's protocol. All the subgroups B1, B2 and B3 were subjected to No heat, 20 seconds and 60 seconds respectively. Heat was applied by a 420-480 nm Light curing device at 0 distance. A universal testing machine was used to measure the shear bond strength for the specimens.

\section{5- Invivo evaluation:}

A total of 20 patients with split mouth design participated in this study. Each patient had two fillings according to the material used (Equia and Zirconomer Improved). A total number of 40 class I carious lesions were involved in this study. Each group was divided into two equal groups (20 teeth) according to the material used. Conventional class I cavities were prepared. All the preparations were of simple class I cavities. Restorations of the teeth were evaluated at the same day, one week, 1 month and 6 months to determine any signs of failure according to the modified USPHS criteria.

\section{Statistical analysis:}

To compare between the performance of Zirconomer and Equia, Fisher's Exact test was used. Shear bond strength data showed normal (parametric) distribution while leakage data showed non-normal (non-parametric) distribution.

For parametric data; two-way ANOVA test was utilized to investigate the influence of material and heat application on shear bond strength. When ANOVA test is significant, Bonferroni's post-hoc test was utilized for pair-wise comparisons For nonparametric data; Kruskal-Wallis test was utilized to study the effect of material and heat application on leakage. Dunn's test was used for pair-wise comparisons. The level of significance was set at $\mathrm{P} \leq 0.05$ in all tests. Statistical analysis was performed with IBM ${ }^{\circledR}$ SPSS ${ }^{\circledR}$ Statistics Version 20 for Windows.

\section{RESULTS}

\section{Microleakage results:}

Effect of heating on microleakage scores of GI as shown in Table 1:

With Equia group; it was found that $20 \mathrm{sec}$. and $60 \mathrm{sec}$. heated subgroups recorded the lowest leakage median score values while non-heated subgroup recorded the highest leakage median score values as shown in Figure (1). The difference between different heating times was statistically non-significant $(\mathrm{p}>0.05)$ as demonstrated by non-parametric Kruskal-Wallis test. 
Table (1): Leakage score results for all GI groups as function of heating time

\begin{tabular}{|c|c|c|c|c|c|c|c|c|c|c|c|}
\hline \multirow{2}{*}{ Variable } & \multicolumn{10}{|c|}{ Heat } & \multirow{3}{*}{$\begin{array}{c}\text { Statistics } \\
\text { Pvalue }\end{array}$} \\
\hline & \multicolumn{3}{|c|}{ No } & \multicolumn{3}{|c|}{$20 \mathrm{sec}}$. & \multicolumn{3}{|c|}{$60 \mathrm{sec}$} & & \\
\hline \multirow{4}{*}{ GI groups } & Statistics & Median & Min. & $\operatorname{Max}$. & Median & Min. & $\operatorname{Max}$. & Median & Min. & $\operatorname{Max}$. & \\
\hline & Equia & 1.67 & 0 & 4 & 1.5 & 0 & 4 & 1.5 & 0 & 4 & $0.752 \mathrm{~ns}$ \\
\hline & Zirconomer & 2 & 1 & 4 & 1.75 & 0 & 3 & 1.67 & 0 & 4 & $0.500 \mathrm{~ns}$ \\
\hline & Fuji & 1.75 & 0 & 3 & 1.5 & 0 & 4 & 1.5 & 0 & 4 & $0.925 \mathrm{~ns}$ \\
\hline Statistics & Pvalue & \multicolumn{3}{|c|}{$0.500 \mathrm{~ns}$} & \multicolumn{3}{|c|}{$0.925 \mathrm{~ns}$} & \multicolumn{3}{|c|}{$0.752 \mathrm{~ns}$} & \\
\hline
\end{tabular}

$(p>0.05) n s ;$ non-significant $;(p<0.05)$ significant

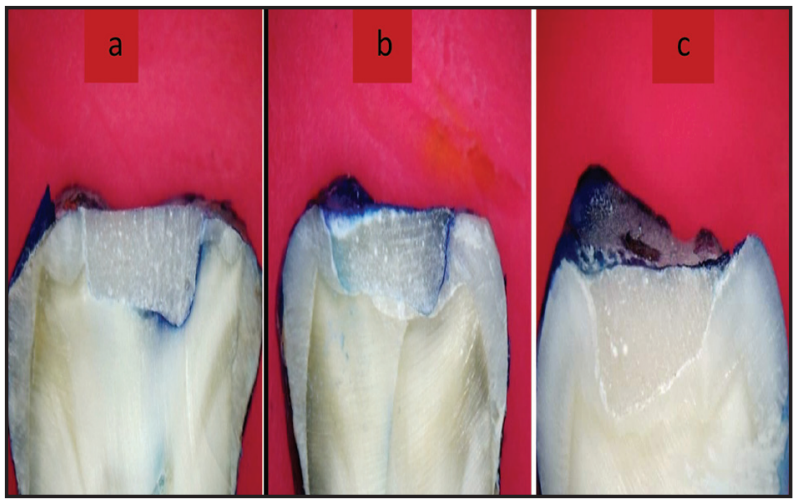

Figure(1) Stereomicroscope images of microleakage of EquiaFort Fil at a) No heat, b) $20 \mathrm{sec}$ of heat application and c) $60 \mathrm{sec}$ of heat application

With Zirconomer group; it was found that 20 sec. and $60 \mathrm{sec}$. heated subgroups recorded the lowest leakage median score values while non-heated subgroup recorded the highest leakage median score values. The difference between different heating times was statistically non-significant ( $p>0.05$ ) as verified by non-parametric Kruskal-Wallis test.

With Fuji group; it was found that $20 \mathrm{sec}$. and $60 \mathrm{sec}$. heated subgroups recorded the same leakage median score values, while non-heated subgroup recorded the highest leakage median score values. The difference between different heating times was statistically non-significant ( $p>0.05)$ as proved by non-parametric Kruskal-Wallis test.

\section{Shear bond strength results:}

With Equia group; the difference between heating times was statistically significant. Pair-wise comparisons between heating times revealed that $60 \mathrm{sec}$. heated subgroup recorded the statistically significantly highest mean shear bond strength (14.9 \pm 3.2$)$. Lower mean shear bond strength was obtained with $20 \mathrm{sec}$. heating time (11.2 \pm 1.2$)$. Nonheated subgroup recorded the statistically significantly lowest mean shear bond strength $(8 \pm 1.8)$.

With Zirconomer group; the difference between heating times was statistically significant. Pairwise comparisons between heating times revealed that $60 \mathrm{sec}$. heated subgroup recorded the statistically significantly highest mean shear bond strength (13.8 \pm 2.3$)$. Lower mean shear bond strength was obtained with $20 \mathrm{sec}$. heating time $(8.4 \pm 1.6)$. Nonheated subgroup recorded the statistically significantly lowest mean shear bond strength $(5.9 \pm 0.6)$.

With Fuji group; the difference between heating times was statistically significant. Pair-wise comparisons between heating times revealed that $60 \mathrm{sec}$. heated subgroup recorded the statistically significantly highest mean shear bond strength (19.7 2 2.6). Lower mean shear bond strength was obtained with 20 sec. heating time $(15.5 \pm 3.3)$. Non-heated subgroup recorded the statistically significantly lowest mean shear bond strength $(11.7 \pm 2.1)$. Effect of heat on shear bond of all GI groups as shown in Table (2). 
Table (2): Shear bond strength results for all GI groups as function of heating time

\begin{tabular}{|c|c|c|c|c|c|c|c|c|}
\hline \multirow{2}{*}{ Variable } & \multicolumn{7}{|c|}{ Heat } & \multirow{3}{*}{$\begin{array}{c}\text { Statistics } \\
\text { Pvalue }\end{array}$} \\
\hline & \multicolumn{2}{|c|}{ No } & \multicolumn{2}{|c|}{20 sec. } & \multicolumn{2}{|c|}{$60 \mathrm{sec}$} & & \\
\hline \multirow{4}{*}{ GI groups } & Statistics & Mean & $S D$ & Mean & $S D$ & Mean & $S D$ & \\
\hline & Equia & $8.0^{\mathrm{CE}}$ & 1.8 & $11.9^{\mathrm{BE}}$ & 1.2 & $14.9^{\mathrm{AE}}$ & 3.2 & $<0.001 *$ \\
\hline & Zirconomer & $5.9^{\mathrm{CF}}$ & 0.6 & $8.4^{\mathrm{BF}}$ & 1.6 & $13.8^{\mathrm{AE}}$ & 2.3 & $<0.001 *$ \\
\hline & Fuji & $11.7^{\mathrm{CD}}$ & 2.1 & $15.5^{\mathrm{BD}}$ & 3.3 & $19.7^{\mathrm{AD}}$ & 2.6 & $<0.001 *$ \\
\hline Statistics & Pvalue & \multicolumn{2}{|c|}{$<0.001^{*}$} & \multicolumn{2}{|c|}{$0.002 *$} & \multicolumn{2}{|c|}{$<0.001^{*}$} & \\
\hline
\end{tabular}

$*(p<0.05)$ significant

$A, B, C$, written in the same row show statistically significant difference between heat applications

$D, E, F$ written in the same column show statistically significant difference between materials

\section{Results of Invivo study:}

The GI restorations were assessed for postoperative sensitivity, color match and secondary caries as shown in tables 3, 4 and 5 respectively. The difference between the restorative materials (EquiaFil Fort and Zirconomer after 6 months showed no statistical significance and demonstrated good clinical performance.

\section{Post-operative sensitivity}

\section{Comparison between the two groups}

At base line as well as after 1 month and 6 months, all cases in the two groups had post-operative sensitivity score (A), so no statistical comparisons between groups could be performed. No statistical significance was noticed between Equia and Zirconomer.

\section{Color mismatch:}

At base line as well as after 1 week, all cases in the two groups had color mismatch predominantly score (B), so no statistical comparisons between groups could be performed. After 1 month as well as 6 months, with minor record (C), no statistically significant difference was found between Equia and Zirconomer.
Table (3): Comparison between post-operative sensitivity scores in the two groups

\begin{tabular}{|c|c|c|c|c|c|}
\hline \multirow[t]{2}{*}{ Time } & \multicolumn{2}{|c|}{$\begin{array}{c}\text { Equia } \\
(\mathrm{n}=20)\end{array}$} & \multicolumn{2}{|c|}{$\begin{array}{l}\text { Zirconomer } \\
(\mathrm{n}=20)\end{array}$} & \multirow[t]{2}{*}{$P$-value } \\
\hline & $\mathrm{N}$ & $\%$ & $\mathrm{n}$ & $\%$ & \\
\hline \multicolumn{5}{|l|}{ Base line } & \multirow[b]{2}{*}{$\mathrm{NC} * *$} \\
\hline Score A & 20 & 100 & 20 & 100 & \\
\hline \multicolumn{5}{|l|}{1 week } & \multirow{4}{*}{0.487} \\
\hline Score A & 18 & 90 & 20 & 100 & \\
\hline Score B & 1 & 5 & 0 & 0 & \\
\hline Score C & 1 & 5 & 0 & 0 & \\
\hline \multicolumn{5}{|l|}{1 month } & \multirow[b]{2}{*}{$\mathrm{NC}^{* *}$} \\
\hline Score A & 20 & 100 & 20 & 100 & \\
\hline \multicolumn{5}{|l|}{6 months } & \multirow[b]{2}{*}{$\mathrm{NC}^{* *}$} \\
\hline Score A & 20 & 100 & 20 & 100 & \\
\hline
\end{tabular}

NC**: Not Computed (variable constant)

Table (4): Comparison between color match scores in the two groups

\begin{tabular}{|c|c|c|c|c|c|}
\hline \multirow{2}{*}{ Time } & \multicolumn{2}{|c|}{$\begin{array}{c}\text { Equia } \\
(\mathrm{n}=20)\end{array}$} & \multicolumn{2}{|c|}{$\begin{array}{l}\text { Zirconomer } \\
(\mathrm{n}=20) \mathrm{nv}\end{array}$} & \multirow{2}{*}{$P$-value } \\
\hline & $\mathrm{N}$ & $\%$ & $\mathrm{~N}$ & $\%$ & \\
\hline \multicolumn{5}{|l|}{ Base line } & \multirow[b]{2}{*}{ NC** } \\
\hline Score B & 20 & 100 & 20 & 100 & \\
\hline \multicolumn{5}{|l|}{1 week } & \multirow[b]{2}{*}{ NC** } \\
\hline Score B & 20 & 100 & 20 & 100 & \\
\hline \multicolumn{5}{|l|}{1 month } & \multirow{3}{*}{1.000} \\
\hline Score B & 20 & 100 & 19 & 95 & \\
\hline Score C & 0 & 0 & 1 & 5 & \\
\hline \multicolumn{5}{|l|}{6 months } & \multirow{3}{*}{1.000} \\
\hline Score B & 19 & 95 & 18 & 90 & \\
\hline Score C & 1 & 5 & 2 & 10 & \\
\hline
\end{tabular}

NC**: Not Computed (variable constant) 


\section{Secondary caries:}

At base line as well as after 1 week, all cases in the two groups had secondary caries score (A), so no statistical comparisons between groups could be performed. After 1 month as well as 6 months, minor record for (B), no statistically significant difference was found between the Equia and Zirconomer.

Table (5): Comparison between secondary caries scores in the two groups

\begin{tabular}{|c|c|c|c|c|c|}
\hline \multirow[t]{2}{*}{ Time } & \multicolumn{2}{|c|}{$\begin{array}{c}\text { Equia } \\
(\mathrm{n}=20)\end{array}$} & \multicolumn{2}{|c|}{$\begin{array}{l}\text { Zirconomer } \\
(\mathrm{n}=20)\end{array}$} & \multirow[t]{2}{*}{$P$-value } \\
\hline & $\mathrm{n}$ & $\%$ & $\mathrm{~N}$ & $\%$ & \\
\hline \multicolumn{5}{|l|}{ Base line } & \multirow[b]{2}{*}{$\mathrm{NC}^{* *}$} \\
\hline Score A & 20 & 100 & 20 & 100 & \\
\hline \multicolumn{5}{|l|}{1 week } & \multirow[b]{2}{*}{$\mathrm{NC}^{* *}$} \\
\hline Score A & 20 & 100 & 20 & 100 & \\
\hline \multicolumn{5}{|l|}{1 month } & \multirow{3}{*}{1.000} \\
\hline Score A & 20 & 100 & 19 & 95 & \\
\hline Score B & 0 & 0 & 1 & 5 & \\
\hline \multicolumn{5}{|l|}{6 months } & \multirow{3}{*}{1.000} \\
\hline Score A & 20 & 100 & 19 & 95 & \\
\hline Score B & 0 & 0 & 1 & 5 & \\
\hline
\end{tabular}

NC**: Not Computed (variable constant)

\section{DISCUSSION}

Microleakage massively causes failure of the restorative materials to a high extent. As a result, there has always been clinical trials to reach maximum adaptation of restorative materials to the internal cavity walls to seal the cavity against the leakage of oral fluids and microorganisms ${ }^{(8)}$. Also, the shear bond strength test is widely used to assess the clinical bonding action of the dental materials, because of the fact that the restorative material of high shear bond resists the high dislodging forces at the tooth restoration interface particularly regarding the GICs, which present low bond strength ${ }^{(3)}$. It is known that the traditional GICs are associated with some disadvantages such as delayed setting reaction, low early strength, and other poor esthetics ${ }^{(9)}$. That's why the conventional GIC has undergone many modifica- tions to overcome these limitations. Also, recent researches have suggested to accelerate the setting of GI, by the heat produced from a light cure unit ${ }^{(10)}$. When the GIC matrix rapidly matures, its early mechanical properties ${ }^{(11)}$ and its marginal adaption are improved ${ }^{(12)}$.

Hence, in the present study the microleakage and the shear bond were investigated of Class I cavities restored with reinforced GI Equia Fort Fil (a highly viscous conventional GIC with a resin coat), Zirconomer (Zirconia + GI) and Fuji II LC (RMGI) after applying of heat at $20 \mathrm{sec}, 60 \mathrm{sec}$.

Shear bond test results revealed that regardless of the heat applied, the RMGIC (Fuji II) recorded statistically significant highest shear bond mean value followed by highly viscous GI with a resin coat (Equia) meanwhile, zirconia filled GI (Zirconomer) recorded statistically significant lowest shear bond mean value. The microleakage results revealed that regardless the heat time, Equiafil fort recorded statically nonsignificant lowest leakage mean value followed by Fuji II LC meanwhile Zirconomer recorded statically nonsignificant the highest leakage mean value.

A study assessed the microleakage of high viscosity GIC and compared it to a Resin modified glass ionomer cement (RMGIC) and then reported that, high viscosity GIC showed significantly lower microleakage scores when compared to RMGIC. They claimed that the resin content in RMGI increased the polymerization shrinkage while GIC had minimal setting shrinkage and approximately one half that of resins ${ }^{(9)}$.

According to a previous research on marginal leakage of Zirconmer and highly viscous GI, the sealing ability of highly viscous GI was much better than Zirconomer. This was thought due to presence of large sized Zirconia particles in Zirconomer that hinders its good adaptation to the tooth surface ${ }^{(13)}$.

Higher shear bond strength values for RMGI were recorded in comparison to viscous GI. The viscous GI bonds to tooth substrate by ion-exchange while RMGIs bond to tooth substrate through both 
ion-exchange and micromechanical interlock (dual mechanism of adhesion), In addition, the good wetting ability of light activated resin hydroxyethyl methacrylate (HEMA) might also increase the bond strength ${ }^{(14)}$. This may explain the higher shear bond strength of high viscous GI with a resin coat (Equia) than zirconia filled GI (Zirconomer) in our study.

In this current study, results revealed that heat had a positive effect on decreasing the microleakage and increasing the shear bond in all groups. These results were in agreement with a previous study where the effect of heat applied on microleakage of GIC was studied and had statistically significant lower leakage values than non heated groups. It was concluded that the marginal adaptation of material on the internal cavity walls was improved and the porosity inside the GIC was decreased upon heating with a polymerization light during its setting ${ }^{(11)}$.

Moreover, other study stated that the early application of heat improved the bond strength. The dental polymerization heat accelerates the chemical reaction of GIC. The earlier the maturation of GIC, the better its mechanical properties in clinical situations ${ }^{(15)}$. On the other hand, a previous study stated that there wasn't any effect of heat on the mechanical properties of GI. This may be due to the difference in the methodology ${ }^{(16)}$.

As for the clinical study, results revealed statically insignificant differences between Equia Fort Fil and Zirconomer regarding the USPHS criteria. Both materials exhibited good clinical outcome over the observation periods (1 week, 1 month and 6 months). A possible explanation for this satisfaction in clinical performance is the fact that all restorations were done in conservative class I cavities, which may be contributed for more effective sealing, reducing marginal leakage. In addition, all patients were instructed to follow oral hygiene measures.

In this study, the difference between the follow up periods was insignificant for color match, secondary caries and post-operative sensitivity. This may be due to the short time of evaluation (6 months). This agreed with a previous study which had assessed the clinical performance of Equia for four years in clinical trial and stated that overall clinical success rate was $100 \%$ for Class I cavities ${ }^{(17)}$.

The lack of postoperative sensitivity after 6 months might be due to the restoration placement techniques and the properties of the restorative materials that decrease the hydrostatic dentin fluid movement. Hypersensitivity might be also due to many other factors, such as microleakage, trauma and other sources ${ }^{(18,19)}$. Furthermore, sensitivity is influenced by the patient, the dentist, the choice of the material and the type of the tooth. The percentage for Alpha score for Equia and Zirconomer was $100 \%(\mathrm{n}=20)$ at 6 months follow up period.

The presence of secondary caries might be due to many reasons such as the type of restoration, clinical procedures and patient's caries index, which may affect their clinical performance. Results of this study revealed insignificant difference in the follow up periods for secondary caries. All restorations of Equia were rated Alpha score at 1 week, 1 month and 6 months while 19 Zirconomer restorations were rated Alpha score (95\%) and 1 restoration was rated Bravo score at 6 months follow up period. This result is in agreement with a study which reported that Equia was not susceptible for secondary caries in restored premolars in vitro ${ }^{(20)}$.

The clinical success obtained by the absence of secondary caries in Equia was probably a result of the adequate restorative technique, short evaluation period and good oral hygiene of the patients. It must be reported that all restorations were placed in the university clinic and the patients were selected with good oral hygiene, low caries risk and without severe malocclusion or bruxism.

With regard to the color match, the color stability of both restorative materials was good. 19 Equia restorations (95\%) were rated Bravo score and 1 restoration (5\%) were rated Charlie score and for Zirconomer 18 restorations (90\%) were rated 
Bravo and 2 restorations (10\%) were rated Charlie at 6 months follow up periods. The color match is thought to be involved with size of glass particles. Also; a previous study reported that Equia was successful regarding color match after a six-year clinical evaluation period ${ }^{(21)}$.

\section{CONCLUSION}

Within the limitations of the present study, it was concluded that:

1. Application of heat improves shear bond strength and microleakage of GICs.

2. The highly viscous GIC restorative system with a resin coat seemed to be promising regarding the shear bond strength and the microleakage rather than the zirconia reinforced GI system.

3. Equia Fort Fil and Zirconomer Improved showed satisfying clinical outcome according to the criteria assessed in Class I.

\section{RECOMMENDATION}

1. Further investigations are needed to study the other aspects of the Equia Fort Fil and Zirconomer Improved restorations to help predicting their clinical success.

2. Heating of GIC is recommended for use as a command set method.

\section{REFERENCES}

1. Lohbauer U. Dental glass-ionomer cements as permanent filling materials? Properties, limitations and future trends. Materials (Basel). 2010; 3: 76-96.

2. Ilie N, Hickel R, Valceaune A, Huth K. Fracture toughness of dental restorative materials. Clin Oral Invest 2012;16:489-98.

3. Makkar S, Chauhan J ,Tamanpreet, Singh S. Comparative Evaluation of microleakage in class II restorations using open sandwich technique with resin modified glass ionomer and Zirconomer as an intermediate material-an invitro study. J. Dent and Med Sci 2016; 15: 78-83.
4. Bonifacio C, Werner A, Kleverlaan C. Coating glass ionomer cements with a nanofilled resin. Acta Odont Scand 2012; 70:471-7.

5. Diem V, Tyas M, Hien C, Phuong L, Khanh N. The effect of a nano-filled resin coating on the 3-year clinical performance of a conventional high-viscosity glass ionomer cement. Clin Oral Invest $2014 ; 18: 753-9$.

6. Fagundes T, Barata T, Bresciani E, Cefaly D, Carvalho C, Navarro M. Influence of ultrasonic setting on tensile bond strength of glass ionomer cements to dentin. J Adhes Dent 2006;8:401-7.

7. Yan Z, Sidhu S, Carrick T, McCabe J: Response to thermal stimuli of glass-ionomer cements. Dent Mater 2007;23:597-600.

8. Abdel Halim S, Zaki D. Comparative evaluation of microleakage among three different glass ionomer types. Oper Dent 2011; 36: 36-42.

9. Upadhyay S, Rao A. Nanoionomer evaluation of microleakage. J Ind Soc Pedod Prev Dent 2011;29:20-4.

10. O'Brien T, Shoja-Assadi F, Lea S, Burke F, Palin W. Extrinsic energy sources affect hardness through depth during set of a glass-ionomer cement. J Dent. 2010;38:49-5.

11. Gorseta K, Glavina D, Skrinjaric I. Influence of ultrasonic excitation and heat application on the microleakage of glass ionomer cements. Aust Dent J. 2012;57:453-7.

12. Gavic L, Gorseta K, Glavina D, Czarnecka B, Nicholson J. Heat transfer properties and thermal cure of glass-ionomer dental cements . J Mater Sci Mater Med. 2015; 26:249.

13. Asafarlal S. Comparative evaluation of microleakage, surface roughness and hardness of three glass ionomer cements -Zirconomer, Fujii IX Extra GC and Ketac Molar: An In vitro study. Dent J 2017, 7: 1-5.

14. Yassen A, Alhussiny H, S.Eldine D. Shear bond strength of viscous versus resin modified glass ionomers to dentin conditioned with different protocols. EDJ. 63: 165-72.

15. Gorseta K, Skrinjaric T, Glavina D. The effect of heating and ultrasound on the shear bond strength of glass ionomer cements. Col Antropol. 2012;4:1307-12.

16. Menne-Happ U, Ilie N. Effect of heat application on the mechanical behavior of glass ionomer cements. Clin Oral Invest. 2014;18:643-50.

17. Gurgan S, Kutuk Z, Ergin E, Oztas S, Cakir F. Four year Randomized clinical trial to evaluate the clinical performance of a glass ionomer restorative system. Opera Dent. 2015, 40:134-43. 
18. Prati C, Cervellati F, Sanasi V, Montebugnoli L. Treatment of cervical dentin hypersensitivity with resin adhesives: 4-week evaluation. Am J Dent. 2001; 14: 378-82.

19. Swift EJ Jr, May KN Jr, Mitchell S. Clinical evaluation of Prime and Bond 2.1 for treating cervical dentin hypersensitivity. Am J Dent. 2001 ;14:13-6.
20. Schwendicke F, Kniess J, Paris S, Blunck U. Margin integrity and secondary caries of lined or non-lined composite and glass hybrid restorations after selective excavation in vitro. Oper Dent. 2017;42:155-64.

21. Türkün L, Kanik Ö. A prospective six-year clinical study evaluating reinforced glass-ionomer cements with resin coating on posterior teeth: Quo Vadis? Oper Dent. 2016;41:587-98. 